\title{
Paeoniflorin influences breast cancer cell proliferation and invasion via inhibition of the Notch-1 signaling pathway
}

\author{
JING ZHANG ${ }^{1}$, KUN YU $^{2}$, XUEDONG HAN ${ }^{3}$, LINLIN ZHEN $^{3}$, MINMIN LIU $^{3}$, \\ XIWEN ZHANG ${ }^{2}$, YI REN ${ }^{3}$ and JIANHUA $\mathrm{SHI}^{3}$ \\ ${ }^{1}$ Department of Breast Surgery, Xuzhou Tumor Hospital, Xuzhou, Jiangsu 221000; Departments of ${ }^{2}$ Cardiology and \\ ${ }^{3}$ Breast and Thyroid Surgery, Huai'an First People's Hospital, Nanjing Medical University, Huai'an, Jiangsu 223300, P.R. China
}

Received April 3, 2016; Accepted May 23, 2017

DOI: $10.3892 / \mathrm{mmr} .2017 .8002$

\begin{abstract}
Breast cancer is one of the most frequently occurring malignant tumors affecting women's health. At least one million new cases are diagnosed each year. Therefore, research that aims to identify strategies that inhibit the growth of breast cancer cells has become a primary worldwide focus. Traditional Chinese medicine (TCM) is regarded as a valuable resource in China, and numerous monomer compositions extracted from TCMs have been demonstrated to exhibit antitumor effects. The present study aimed to determine the impact of paeoniflorin (PF) on breast cancer cell proliferation and invasion, and to explore the mechanisms underlying its effects. Different concentrations of PF were applied to MCF-7 cells at various time points and the Cell Counting kit- 8 assay was used to determine cell proliferation, a transwell invasion assay was employed to determine cell invasion, reverse transcription-polymerase chain reaction was used to determine notch homolog-1 (NOTCH-1) and Hes family basic helix-loop helix transcription factor (HES)-1 mRNA expression levels, and western blotting was used to determine NOTCH-1 and HES-1 protein expression levels. The results demonstrated that PF inhibited the proliferation of MCF-7 cells in a dose- and time-dependent manner. Following treatment with different concentrations of PF, the total number of cells present in the PF-treated groups was significantly lower when compared with the untreated control group $(\mathrm{P}<0.05)$. With increasing doses of PF, the rate of cell invasion significantly decreased, indicating a dose-dependent association. NOTCH-1 and HES-1 mRNA expression levels were reduced when compared with the untreated control group, which reached a statistical significance following treatment with 15 and $30 \mu \mathrm{M} \mathrm{PF}(\mathrm{P}<0.05)$. NOTCH-1
\end{abstract}

Correspondence to: Dr Jianhua Shi or Dr Yi Ren, Department of Breast and Thyroid Surgery, Huai'an First People's Hospital, Nanjing Medical University, 6 Beijing West Road, Huai'an, Jiangsu 223300, P.R. China

E-mail: j_h_shi0331@163.com

E-mail: renyiRY19@163.com

Key words: paeoniflorin, notch homolog-1, cell proliferation, cell invasion and HES-1 protein levels demonstrated a similar trend to the mRNA levels, whereby an increase in the concentration of PF was associated with a decrease in NOTCH-1 and HES-1 protein expression levels. The results of the present study therefore suggest that PF may inhibit the proliferation and invasiveness of breast cancer cells via inhibition of the NOTCH-1 signaling pathway.

\section{Introduction}

Breast cancer is one of the most frequently occurring malignant tumors affecting women worldwide (1). The incidence of breast cancer is increasing, with a demonstrated younger age of onset in recent years (2). Each year $>1$ million cases are newly diagnosed, and there are $>400,000$ cases of mortality (3). The incidence rate of breast cancer in China is increasing, and it is currently the most frequently occurring malignant tumor in females. Metastasis is the final stage of development in breast cancer and is the leading cause of mortality in patients. Therefore, identifying strategies that inhibit breast cancer cell growth, invasion and metastasis are critical for the successful treatment of breast cancer, and are the focus of primary research. Recent studies have facilitated the development of more effective therapeutic strategies for breast cancer treatment in areas including surgery, chemotherapy, radiotherapy, endocrine therapy and targeted molecular treatment (4-8). These have resulted in an increase in the curative effects and survival rates of breast cancer patients to varying degrees (4-8). At present, the primary treatment for the inhibition of breast cancer metastasis is chemotherapy; however, the results are generally unsatisfactory. In addition, currently available chemotherapeutic agents are unable to effectively distinguish tumor cells from healthy human cells, which leads to adverse and toxic side effects. Therefore, the identification of a highly potent antitumor treatment with low toxicity is a primary focus of research.

The use of traditional Chinese medicine (TCM) as a therapeutic strategy for various diseases is of great value in China. It has been demonstrated previously that numerous monomer compositions extracted from TCMs exhibit antitumor effects, such as curcumin (9) and puerarin (10). Paeoniflorin (PF) was first isolated from the peony plant (Ranunculaceae family) in 1963, and is the primary active monomer component in peonies. $\mathrm{PF}$ is a monoterpene glycoside compound that 
exhibits anti-inflammatory, immune regulatory and neuroprotective properties (11-13). In addition, previous studies have demonstrated that that PF may exhibit antitumor effects, including inhibitory effects on the proliferation, invasion and metastasis of lung (14), gastric (15), liver (16) and cervical cancer cells (17). Zhang et al (18) revealed that PF inhibited the proliferation and invasion of breast cancer cells by inhibiting the Notch-1 signaling pathway.

The aim of the present study was to investigate the effects of PF on the MCF-7 breast cancer cell line. Cells were treated with increasing concentrations of $\mathrm{PF}$ for different durations in order to examine the effects of PF on their proliferation and invasion rates. It has been previously demonstrated that silencing of notch homolog-1 (NOTCH-1) decreases the invasive ability of breast cancer cells (19), and inhibition of the expression of NOTCH-1 inhibits breast cancer cell proliferation (20). These results suggest that the NOTCH-1 signaling pathway may be important in the regulation of breast cancer cell proliferation and invasion. Therefore, the present study examined the expression levels of key genes of the NOTCH-1 signaling pathway, in order to identify the potential mechanisms underlying the effect of PF on breast cancer cells.

\section{Materials and methods}

Instrumentsandreagents. The MCF-7 breast cancercell line was obtained from American Type Culture Collection (Manassas, VA, USA). RPMI-1640 culture medium, bovine serum albumin (BSA) and fetal bovine serum (FBS) was obtained from Gibco; Thermo Fisher Scientific, Inc. (Waltham, MA, USA). $\mathrm{PF}$ was purchased from the National Institute for the Control of Pharmaceutical and Biological Products (Beijing, China). The Cell Counting kit (CCK)-8 assay was purchased from Peptide Institute, Inc. (Osaka, Japan), the transwell chamber system was obtained from Corning Incorporated (Corning, NY, USA), the Nanodrop2000 nucleic acid protein detector was purchased from Thermo Fisher Scientific, Inc., the RNA extraction reagent, TRIzol ${ }^{\circledR}$, was purchased from Invitrogen; Thermo Fisher Scientific, Inc., the SYBR Green Mixture was purchased from Toyobo Life Science (Osaka, Japan), and the real-time fluorescence quantitative reverse transcription-quantitative polymerase chain reaction (RT-qPCR) kit was obtained from Takara Bio, Inc., (Otsu, Japan). The gel imaging system and the ViiA7 Real-Time PCR System were obtained from Applied Biosystems; Thermo Fisher Scientific Inc., the total protein extraction kit was purchased from BestBio (Shanghai, China), and the Coomassie Brilliant Blue protein assay kit was obtained from Shanghai Meiji Biological Technology Co. Ltd. (Shanghai, China). In addition, the SDS-PAGE gel, phosphate-buffered saline plus $0.1 \%$ Tween-20 (PBST) solution (Nanjing Senbeijia Biological Technology Co., Ltd., Nanjing, China), vertical polyacrylamide electrophoresis apparatus and the GIS-2020D gel imaging analysis system were purchased from Sigma-Aldrich; Merck KGaA (Darmstadt, Germany). Antibodies for NOTCH-1 (cat. no. ab52627), Hes family basic helix-loop helix transcription Factor (HES)-1 (cat. no. ab108937), $\beta$-actin (cat. no. ab8226) and horseradish peroxidase (HRP)-conjugated goat anti-rabbit secondary antibody (cat. no. ab6721) were purchased from Abcam (Cambridge, MA, USA).
MCF-7 cell culture. The MCF-7 cells were cultured in RPMI-1640 medium supplemented with streptomycin and penicillin $(1,000 \mathrm{IU} / \mathrm{l})$ and $10 \% \mathrm{FBS}$. The cells were then placed in an incubator at $37^{\circ} \mathrm{C}$ with $5 \% \mathrm{CO}_{2}$, and the cells were passaged once every 2-3 days. During passaging, the culture medium in the culture dish was discarded and the cells were washed with PBS twice. Following incubation of cells with trypsin for $1 \mathrm{~min}$, the adherent cells were gently pipetted into single cell suspensions, which were then centrifuged at $500 \mathrm{xg}$ at room temperature for $5 \mathrm{~min}$. The supernatants were subsequently discarded and the cell pellet was re-suspended in $1 \mathrm{ml}$ medium; $200 \mu \mathrm{l}$ of cell suspension was used for subculture.

CCK-8 cell proliferation assay. Cells growing in the logarithmic phase were digested and counted, then $1 \times 10^{4}$ cells/well were transferred to a 96-well plate. The cells were allowed to adapt to the culture environment for $1 \mathrm{~h}$, before they were treated with $0,7.5,15$ and $30 \mu \mathrm{M}$ PF for 24,48 and $72 \mathrm{~h}$. For each dose, there were five replicate wells, and a blank control group was included in each assay. Following the treatment intervention, the cells were washed with PBS three times before $100 \mu$ l CCK- 8 mixture (1:10, CCK- 8 reagent: medium) was added to the wells, which were then incubated in a $5 \%$ $\mathrm{CO}_{2}$ incubator at $37^{\circ} \mathrm{C}$ for $2 \mathrm{~h}$. The absorbance (A) values were measured at a wavelength of $450 \mathrm{~nm}$ using a microplate reader, and the rate of cell survival was calculated using the following formula: Cell survival rate $=\left(A_{\text {treated group }}-A_{\text {blank control group }}\right) /\left(A_{\text {control }}\right.$ group $\left.-\mathrm{A}_{\text {blank control group }}\right)$.

Transwell invasion assay. A total of $50 \mathrm{mg} / 1$ Matrigel was diluted in serum-free medium at a ratio of $1: 8$. The inner surface of the membrane at the bottom of the transwell chamber was coated with the Matrigel matrix mixture, and the chamber was placed in an incubator for $30 \mathrm{~min}$ at $37^{\circ} \mathrm{C}$ before it was transferred to a safety cabinet for air-drying overnight. The chamber was blocked with serum-free RPMI-1640 medium containing $10 \mathrm{~g} / \mathrm{l} \mathrm{BSA}$ for $30 \mathrm{~min}$. MCF-7 cells were serum-starved for $24 \mathrm{~h}$ before they were digested, collected and centrifuged at $500 \mathrm{x}$ g for $5 \mathrm{~min}$ at room temperature. The supernatant was discarded and the cell pellet was washed with PBS twice before the cells were re-suspended in serum-free RPMI-1640 medium containing $10 \mathrm{~g} / 1 \mathrm{BSA}$. The cell concentration was adjusted to $5 \times 10^{7}$ cells $/ 1$. A total of $200 \mu \mathrm{l}$ cell suspension was added into the lower transwell chamber, and $600 \mu$ RPMI-1640 culture medium supplemented with $10 \%$ FBS was added into the lower chamber. Cells were incubated for $6 \mathrm{~h}$ to allow them to adapt to the culture conditions before $\mathrm{PF}$ was added to the lower chamber at concentrations of $0,7.5$, 15 and $30 \mu \mathrm{M}$. A total of 5 replicate wells for each group were analyzed, and each assay included a blank control. Following the addition of $\mathrm{PF}$, the plates were incubated for $48 \mathrm{~h}$ at $37^{\circ} \mathrm{C}$. Cells that remained in the upper chamber of the transwell invasion system were removed using a cotton swab, and the wells were washed with PBS twice. The cells on the underside of the upper chamber were fixed with methanol (100\%) for $15 \mathrm{~min}$ at room temperature, stained with $1 \%$ crystal violet for $10 \mathrm{~min}$ at room temperature, and then observed under an inverted microscope for analysis and counting; for each group a total of five fields were analyzed under the 400 -fold microscope. A total of three independent repeats of this assay were performed. 
Detection of NOTCH-1 and HES-1 mRNA expression levels. Following treatment of MCF-7 $\left(5 \times 10^{5}\right)$ cells with $0,7.5,15$ and $30 \mu \mathrm{M}$ PF for $48 \mathrm{~h}$, the cells were collected and RNA was extracted using TRIzol ${ }^{\circledR}$ reagent according to the manufacturer's instructions. The purity and content of RNA was determined using the Nanodrop 2000 protein and nucleic acid analyzer. The integrity of RNA was detected via $1 \%$ agarose gel electrophoresis. A total of $1 \mu \mathrm{g}$ RNA was reverse transcribed to cDNA using an RNA reverse transcription kit (Takara Bio, Inc.), according to the manufacturer's protocol. The reaction mixture for the quantitative (q)PCR reaction consisted of the following: $5 \mu 1$ SYBR Green Mixture (2X), $0.5 \mu \mathrm{l}$ cDNA, $0.5 \mu \mathrm{l}$ forward primer

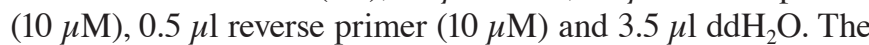
thermocycling conditions for the $\mathrm{qPCR}$ reaction were as follows: Pre-denaturation at $95^{\circ} \mathrm{C}$ for $10 \mathrm{~min}$, followed by 40 cycles of denaturation at $95^{\circ} \mathrm{C}$ for $15 \mathrm{sec}$ and annealing and extension at $60^{\circ} \mathrm{C}$ for $60 \mathrm{sec}$. The following primers were used: Notch-1, forward, 5'-CACTGTGGGCGGGTCC-3' and reverse, 5'-GTT GTATTGGTTCGGCACCAT-3; HES-1, forward, 5'-TAGCTC GCGGCATTCCAAGC-3' and reverse, 5'-GTGCTCAGCGCA GCCGTCATCT-3'; $\beta$-actin, forward, 5'-CCACACTGTGCC CATCTACG-3' and reverse, 5'-AGGATCTTCATGAGGTAG TCAGTCAG-3'. This was performed using the ViiA7 Real-Time PCR System. Experiments were performed in triplicate for each group and $\beta$-actin was used as the internal reference gene. The relative expression level of mRNA was determine using the by $2^{-\Delta \Delta C q}$ method (21).

Detection of NOTCH-1 and HES-1 protein expression levels. Following treatment of MCF-7 $\left(5 \times 10^{5}\right)$ cells with $0,7.5,15$, and $30 \mu \mathrm{M}$ PF for $48 \mathrm{~h}$, the cells were lysed in lysis buffer $(20 \mathrm{mM}$ Tris- $\mathrm{HCl}, \mathrm{pH} 8.0,100 \mathrm{mM} \mathrm{KCl}$ and $0.2 \mathrm{mM}$ EDTA) and centrifuged at 2,600 x g for $30 \mathrm{~min}$ at room temperature. Total proteins were then extracted from the supernatant using the BestBio kit. The protein concentration was determined using the Coomassie Brilliant Blue protein assay kit, and the buffers were added into protein solutions and boiled. Protein samples $(40 \mu \mathrm{g})$ were loaded onto a 6-12\% SDS-PAGE gel and electrophoresed. Following the addition of transfer buffers, the gel was transferred to a nitrocellulose membrane. The membrane was then blocked with PBST containing 5\% skim milk powder and placed on a shaker at room temperature for $2 \mathrm{~h}$. PBST was used to wash the membrane three times before the primary antibodies were added and incubated on a shaker in the refrigerator at $4^{\circ} \mathrm{C}$ overnight. The following primary antibodies were used: NOTCH-1 $(1: 1,000)$, HES-1 (1:2,000) and $\beta$-actin $(1: 2,000)$. The membrane was then washed with PBST for $30 \mathrm{~min}$ and incubated for $1 \mathrm{~h}$ at the room temperature with the HRP-conjugated goat anti-rabbit secondary antibody $(1: 10,000)$. Following washing with PBST three times, the electroluminescence detection reagent (Thermo Fisher Scientific, Inc.) was evenly spread on the nitrocellulose membrane. The optical densities of NOTCH-1, HES-1 and $\beta$-actin protein bands were analyzed using the GIS-2020D gel imaging analysis system, and the ratio of NOTCH-1 and HES-1 protein band optical densities were compared with the $\beta$-actin protein band optical density to determine the expression intensities of the NOTCH-1 and HES-1 proteins.

Statistical analysis. SPSS software (version, 13.0; SPSS, Inc., Chicago, IL, USA) was used to perform statistical analysis, and
Table I. Effect of paeonifiorin on MCF-7 cell proliferation.

\begin{tabular}{lccc}
\hline & \multicolumn{3}{c}{ Time $(\mathrm{h})$} \\
\cline { 2 - 4 } $\begin{array}{l}\text { Concentration } \\
\mu \mathrm{M})\end{array}$ & 24 & \multicolumn{3}{c}{48} & 72 \\
\hline 0 & $1.00 \pm 0.042$ & $1.00 \pm 0.043$ & $1.00 \pm 0.041$ \\
7.5 & $0.89 \pm 0.038^{\mathrm{a}}$ & $0.81 \pm 0.038^{\mathrm{a}, \mathrm{b}}$ & $0.74 \pm 0.040^{\mathrm{a}-\mathrm{c}}$ \\
15 & $0.80 \pm 0.041^{\mathrm{a}}$ & $0.73 \pm 0.039^{\mathrm{a}, \mathrm{b}}$ & $0.61 \pm 0.044^{\mathrm{a}-\mathrm{c}}$ \\
30 & $0.71 \pm 0.043^{\mathrm{a}}$ & $0.65 \pm 0.041^{\mathrm{a}, \mathrm{b}}$ & $0.52 \pm 0.037^{\mathrm{a}-\mathrm{c}}$ \\
\hline
\end{tabular}

${ }^{\mathrm{a}} \mathrm{P}<0.05$ vs. $0 \mu \mathrm{M}$-treated group; ${ }^{\mathrm{b}} \mathrm{P}<0.05$ vs. $24 \mathrm{~h}$; ${ }^{\mathrm{C}} \mathrm{P}<0.05$ vs. $48 \mathrm{~h}$.

normally distributed data are presented as the mean \pm standard deviation. One-way analysis of variance (ANOVA) was used to measure the effect of PF on cell invasion capacity and the mRNA expression levels of NOTCH1 and HES-1. Repeated measures ANOVA was used to analyze the effect of PF on the proliferation of MCF-7 cells at different time points, and Mauchly's sphericity test was used to validate the results. The post hoc Dunnett's test was used for multiple comparisons. GraphPad Prism 5.0 software (GraphPad Software, Inc., La Jolla, CA, USA) was used to generate the graphs. $\mathrm{P}<0.05$ was considered to indicate a statistically significant difference.

\section{Results}

PF inhibits cell proliferation. MCF-7 cells were treated with increasing concentrations of $\mathrm{PF}$ at various time points, and the effect of PF on the proliferation of MCF-7 cells was determined using the CCK-8 assay. The results of the repeated measures ANOVA test indicated that statistically significant differences among all time points were observed $(\mathrm{F}=600.02$; $\mathrm{P}<0.05)$. In addition, it was revealed that $\mathrm{PF}$ significantly inhibited the proliferation of MCF-7 cells in a time- and concentration-dependent manner $(\mathrm{F}=100.93$; $\mathrm{P}<0.05$; Table I). $\mathrm{PF}$ was observed to inhibit the proliferation of MCF-7 cells in a dose- and time-dependent manner (Table I. The inhibitory effect of $30 \mu \mathrm{M}$ PF on the proliferation of cells was most pronounced when the cells were treated for $72 \mathrm{~h}$.

PF inhibits cell invasion capacity. MCF-7 cells were treated for $48 \mathrm{~h}$ with $0,7.5,15$ and $30 \mu \mathrm{M} \mathrm{PF}$, and the transwell assay system was used to detect the invasion ability of the cells. The number of cells that traversed the membrane was $132.44 \pm 6.58$ in the control group, whereas $102.56 \pm 5.74,80.72 \pm 7.82$ and $62.46 \pm 6.88$ cells were observed to traverse the membrane following treatment with $7.5,15$ and $30 \mu \mathrm{M} \mathrm{PF}$, respectively (Fig. 1). The results demonstrated that a statistically significant and dose-dependent reduction in cell invasion capabilities was observed in PF-treated cells when compared with control cells ( $\mathrm{F}=99.51 ; \mathrm{P}<0.05$; Fig. 1).

PF decreases NOTCH-1 and HES-1 mRNA expression levels. The $0 \mu \mathrm{M}$ PF-treated group served as a control, and the $\beta$-actin gene served as an internal reference gene for the analysis of NOTCH-1 and HES- 1 mRNA levels. Following treatment with PF for $48 \mathrm{~h}$, the mRNA levels of NOTCH-1 and HES-1 


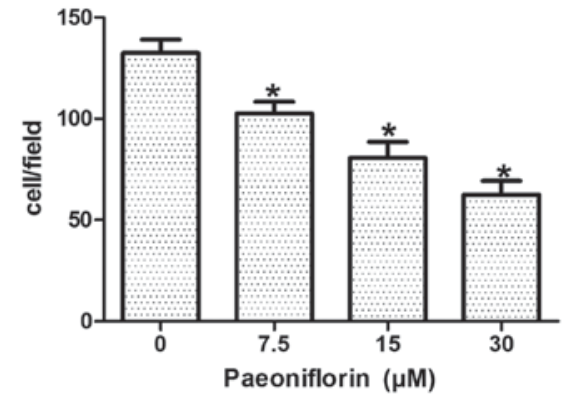

Figure 1. Effect of paeoniflorin on cell invasion capacity. The invasive ability of MCF-7 breast cancer cells treated with $0,7.5,15$ and $30 \mu \mathrm{M}$ paeoniflorin for $48 \mathrm{~h}$, as determined using a transwell migration assay. "P $<0.05$ vs. $0 \mu \mathrm{M}$ paeoniflorin-treated group.

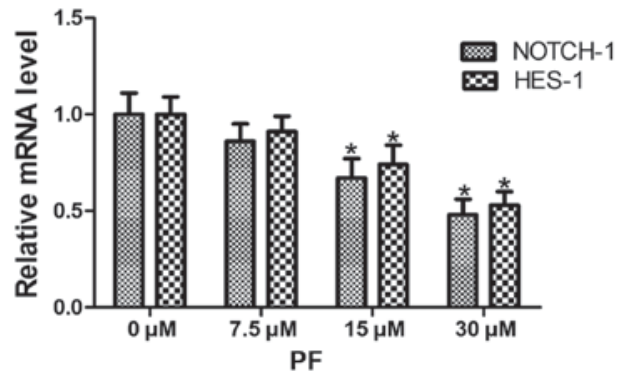

Figure 2. Effect of PF on NOTCH-1 and HES-1 mRNA expression levels. The mRNA expression levels of NOTCH-1 and HES-1 were detected by reverse transcription-polymerase chain reaction following treatment of MCF-7 cells with $0,7.5,15$ and $30 \mu \mathrm{M}$ PF for $48 \mathrm{~h}$. ${ }^{*} \mathrm{P}<0.05$ vs. $0 \mu \mathrm{M}$ PF-treated group. $\mathrm{PF}$, paeoniflorin; NOTCH-1, notch homolog-1; HES-1, Hes family basic helix-loop helix transcription factor.

were determined using RT-qPCR. Different concentrations of PF significantly decreased NOTCH-1 and HES-1 mRNA expression (NOTCH-1, F=29.13, $\mathrm{P}<0.05$; HES-1, $\mathrm{F}=29.97$, $\mathrm{P}<0.05$ ). When compared with the results of the control group, the mRNA expression levels NOTCH-1 and HES-1 decreased in a dose-dependent manner, and were significantly different in the 15 and $30 \mu \mathrm{M}$ PF groups $(\mathrm{P}<0.05)$. No significant differences were observed between treatment with $7.5 \mu \mathrm{M}$ PF and the control (0 $\mu \mathrm{M}$ PF) group (Fig. 2).

PF decreases NOTCH-1 and HES-1 protein expression levels. Following treatment of MCF-7 cells with $0,7.5,15$ and $30 \mu \mathrm{M}$ $\mathrm{PF}$ for $48 \mathrm{~h}$, the protein expression levels of NOTCH-1 and HES-1 were detected via western blotting. The results are presented in Table II and Fig. 3. The pattern of NOTCH-1 and HES-1 protein expression following PF treatment was similar to the pattern of mRNA expression. The protein expression levels of NOTCH-1 and HES-1 decreased with the increasing concentrations of PF (Table II; Fig. 3). A statistically significant difference in NOTCH-1 $(\mathrm{t}=22.15 ; \mathrm{P}<0.05)$ and HES-1 $(\mathrm{t}=19.81$; $\mathrm{P}<0.05)$ protein expression was observed at all concentrations (7.5, 15 and $30 \mu \mathrm{M})$ of PF tested when compared with the untreated controls ( $0 \mu \mathrm{M}$ PF; Table II).

\section{Discussion}

At present, the primary cause of mortality in breast cancer is the recurrence and metastasis of tumors. Therefore, inhibiting
Table II. Effect of paeoniflorin treatment on HES-1 and NOTCH-1 protein expression levels.

\begin{tabular}{lcccc}
\hline & \multicolumn{4}{c}{ Paeoniflorin concentration $(\mu \mathrm{M})$} \\
\cline { 2 - 5 } Protein & 0 & 7.5 & 15 & 30 \\
\hline NOTCH-1 & $1.12 \pm 0.06$ & $0.98 \pm 0.05^{\mathrm{a}}$ & $0.61 \pm 0.07^{\mathrm{a}}$ & $0.55 \pm 0.04^{\mathrm{a}}$ \\
HES-1 & $1.08 \pm 0.08$ & $0.94 \pm 0.07^{\mathrm{a}}$ & $0.79 \pm 0.06^{\mathrm{a}}$ & $0.56 \pm 0.05^{\mathrm{a}}$ \\
\hline
\end{tabular}

${ }^{\text {a }}<0.05$ vs. $0 \mu \mathrm{M}$-treated group. HES-1, Hes family basic helix-loop helix transcription factor; NOTCH-1, notch homolog-1.

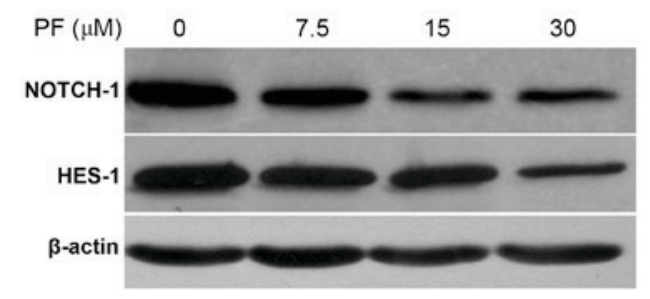

Figure 3. Effect of PF on NOTCH-1 and HES-1 protein expression. The protein expression levels of NOTCH-1 and HES-1 were detected by western blotting following treatment of MCF-7 cells with $0,7.5,15$ and $30 \mu \mathrm{M}$ PF for 48 h. PF, paeoniflorin; NOTCH-1, notch homolog-1; HES-1, Hes family basic helix-loop helix transcription factor.

the invasion and metastasis of cancer cells is of great importance. Current strategies that inhibit breast cancer metastasis predominantly involve chemotherapeutic agents; however, the low specificity of this procedure results in adverse and toxic side effects. Medicinal plants used for the treatment of tumors exhibit few toxic side effects, and may therefore be a more effective treatment when compared with typical chemotherapy drugs $(22,23)$. Numerous studies have demonstrated that PF exhibits antitumor effects $(24,25)$, and may be used for the treatment of cervical, lung and gastric cancers. However, the role of PF in breast cancer remains to be elucidated. Therefore, the present study investigated the role of PF in the inhibition of MCF-7 breast cancer cell growth and invasion in vitro.

The results of the present study demonstrated that increasing concentrations and durations of treatment with $\mathrm{PF}$ were associated with a decrease in the survival rate of MCF-7 cells. The inhibitory effect of PF on MCF-7 cells was most significant following treatment with $30 \mu \mathrm{M} P F$ for $72 \mathrm{~h}$, and the rate of growth inhibition was $\sim 52 \%$. Therefore, PF demonstrated a significant inhibitory effect on the proliferation of MCF-7 cells, in a dose- and time-dependent manner. In addition, following treatment of MCF-7 cells with PF for $48 \mathrm{~h}$, cell invasion decreased with an increasing dose, which suggests that PF successfully inhibited the invasive ability of MCF-7 cells. Zhang et al (18) suggested that the proliferation and invasion of MCF-7 and MDA-MB-231 breast cancer cells are inhibited by $\mathrm{PF}$ at concentrations of 10,20 and $40 \mu \mathrm{M}$, which is consistent with the results of the present study. Therefore, the results of the current study together with those of Zhang et al (18), provide a theoretical foundation for the future application of $\mathrm{PF}$ in the treatment of breast cancer. 
The NOTCH signaling pathway is a conserved signal transduction pathway, which is important for cell development $(26,27)$ and apoptosis $(28,29)$. Out of the four mammalian NOTCH receptors, NOTCH-1 is the primary receptor (30). It has previously been demonstrated that the NOTCH-1 signaling pathway is associated with the proliferation, invasion and metastasis of breast cancer cells $(20,31,32)$; therefore, inhibition of this pathway may subsequently inhibit these characteristics of breast cancer cells. The authors of the present study investigated the potential role of the NOTCH signaling pathway in the inhibition of breast cancer cell proliferation and invasion following treatment with PF. Following exposure of MCF-7 cells to PF for $48 \mathrm{~h}$, the mRNA and protein expression levels of NOTCH-1 and HES-1, which are involved in the NOTCH-1 signaling pathway, were significantly decreased in a dose-dependent manner. The results indicated that PF inhibited the proliferation and invasion of breast cancer cells potentially via inhibition of NOTCH-1 signal transduction. Zhang et al (18) revealed that when MDA-MB-231 breast cancer cells were treated with PF for $48 \mathrm{~h}$ at concentrations of 10,20 and $40 \mu \mathrm{M}$, the mRNA and protein expression levels of NOTCH-1 were significantly reduced. In addition, when the NOTCH-1 gene was silenced, the proliferation of breast cancer cells was suppressed (18). The results of the study by Zhang et al (18) together with the results of the present study, confirmed that the proliferation and invasion abilities of breast cancer cells are inhibited by PF, and that inhibition of the NOTCH-1 signaling pathway may be involved.

In conclusion, the present study provided further evidence to demonstrate that PF inhibits the proliferation and invasion of breast cancer cells via inhibition of NOTCH-1 signal transduction. However, the mechanisms underlying these effects may be more complex. Further investigation is required to elucidate the precise mechanism by which PF mediates inhibition of breast cancer cell growth and invasion, in order to provide a theoretical basis for the application of PF in the clinical treatment of breast cancer.

\section{References}

1. Hortobagyi GN, de la Garza Salazar J, Pritchard K, Amadori D, Haidinger R, Hudis CA, Khaled H, Liu MC, Martin M, Namer M, et al: The global breast cancer burden: Variations in epidemiology and survival. Clin Breast Cancer 6: 391-401, 2005.

2. Forouzanfar MH, Foreman KJ, Delossantos AM, Lozano R, Lopez AD, Murray CJ and Naghavi M: Breast and cervical cancer in 187 countries between 1980 and 2010: A systematic analysis. Lancet 378: 1461-1484, 2011.

3. Coughlin SS and Ekwueme DU: Breast cancer as a global health concern. Cancer Epidemiol 33: 315-318, 2009.

4. Zafrakas M, Papasozomenou P and Emmanouilides C: Sorafenib in breast cancer treatment: A systematic review and overview of clinical trials. World J Clin Oncol 7: 331-336, 2016.

5. Zeichner SB, Terawaki $\mathrm{H}$ and Gogineni K: A review of systemic treatment in metastatic triple-negative breast cancer. Breast Cancer (Auckl) 10: 25-36, 2016.

6. Gao J and Swain SM: Pertuzumab for the treatment of breast cancer: A safety review. Expert Opin Drug Saf 15: 853-863, 2016.

7. Castaneda SA and Strasser J: Updates in the treatment of breast cancer with radiotherapy. Surg Oncol Clin N Am 26: 371-382, 2017.

8. Kaplan HG, Malmgren JA and Atwood MK: Triple-negative breast cancer in the elderly: Prognosis and treatment. Breast J, 2017 Doi: 10.1111/tbj.12813.

9. Huang YT, Lin YW, Chiu HM and Chiang BH: Curcumin induces apoptosis of colorectal cancer stem cells by coupling with CD44 marker. J Agric Food Chem 64: 2247-2253, 2016.
10. Zhang WG, Liu XF, Meng KW and Hu SY: Puerarin inhibits growth and induces apoptosis in SMMC-7721 hepatocellular carcinoma cells. Mol Med Rep 10: 2752-2758, 2014.

11. Kim ID and Ha BJ: Paeoniflorin protects RAW 264.7 macrophages from LPS-induced cytotoxicity and genotoxicity. Toxicol In Vitro 23: 1014-1019, 2009.

12. Gu X, Cai Z, Cai M, Liu K, Liu D, Zhang Q, Tan J and Ma Q: Protective effect of paeoniflorin on inflammation and apoptosis in the cerebral cortex of a transgenic mouse model of Alzheimer's disease. Mol Med Rep 13: 2247-2252, 2016.

13. Zhong SZ, Ge QH, Li Q, Qu R and Ma SP: Peoniflorin attentuates Abeta((1-42))-mediated neurotoxicity by regulating calcium homeostasis and ameliorating oxidative stress in hippocampus of rats. J Neurol Sci 280: 71-78, 2009.

14. Wu Q, Chen GL, Li YJ, Chen Y and Lin FZ: Paeoniflorin inhibits macrophage-mediated lung cancer metastasis. Chin J Nat Med 13: 925-932, 2015.

15. Zheng YB, Xiao GC, Tong SL, Ding Y, Wang QS, Li SB and Hao ZN: Paeoniflorin inhibits human gastric carcinoma cell proliferation through up-regulation of microRNA-124 and suppression of PI3K/Akt and STAT3 signaling. World J Gastroenterol 21: 7197-7207, 2015.

16. Lu JT, He W, Song SS and Wei W: Paeoniflorin inhibited the tumor invasion and metastasis in human hepatocellular carcinoma cells. Bratisl Lek Listy 115: 427-433, 2014.

17. Zhang $\mathrm{L}$ and Zhang S: Modulating Bcl-2 family proteins and caspase-3 in induction of apoptosis by paeoniflorin in human cervical cancer cells. Phytother Res 25: 1551-1557, 2011.

18. Zhang Q, Yuan Y, Cui J, Xiao T and Jiang D: Paeoniflorin inhibits proliferation and invasion of breast cancer cells through suppressing Notch-1 signaling pathway. Biomed Pharmacother 78: 197-203, 2016.

19. Li L, Zhang J, Xiong N, Li S, Chen Y, Yang H, Wu C, Zeng H and Liu Y: Notch-1 signaling activates NF-kappaB in human breast carcinoma MDA-MB-231 cells via PP2A-dependent AKT pathway. Med Oncol 33: 33, 2016.

20. Pei J and Wang B: Notch-1 promotes breast cancer cells proliferation by regulating LncRNA GAS5. Int J Clin Exp Med 8: 14464-14471, 2015.

21. Livak KJ and Schmittgen TD: Analysis of relative gene expression data using real-time quantitative PCR and the 2(-Delta DeltaC(T) method. Methods 25: 402-408, 2001.

22. Xia X, Cole SPC, Cai T and Cai Y: Effect of traditional Chinese medicine components on multidrug resistance in tumors mediated by P-glycoprotein. Oncol Lett 13: 3989-3996, 2017.

23. Jiao L, Dong C, Liu J, Chen Z, Zhang L, Xu J, Shen X, Che J, Yang Y, Huang H, et al: Effects of Chinese medicine as adjunct medication for adjuvant chemotherapy treatments of non-small cell lung cancer patients. Sci Rep 7: 46524, 2017.

24. Zhang LL, Zhang SL and Wang SZ: Relevant study on apoptosis of cervical cancer HeLa cells induced by paeoniflorin. Zhonghua Yi Xue Za Zhi 90: 3371-3375, 2010 (In Chinese).

25. Wu H, Li W, Wang T, Shu Y and Liu P: Paeoniflorin suppress NF-kappaB activation through modulation of I kappaB alpha and enhances 5-fluorouracil-induced apoptosis in human gastric carcinoma cells. Biomed Pharmacother 62: 659-666, 2008.

26. Li XY, Zhai WJ and Teng CB: Notch Signaling in pancreatic development. Int J Mol Sci 17: E48, 2015.

27. Duval F, Mathieu M and Labrecque N: Notch controls effector CD8+ T cell differentiation. Oncotarget 6: 21787-21788, 2015.

28. Dang TP: Notch, apoptosis and cancer. Adv Exp Med Biol 727; 199-209, 2012

29. Ding Y and Shen Y: Notch increased vitronection adhesion protects myeloma cells from drug induced apoptosis. Biochem Biophys Res Commun 467: 717-722, 2015.

30. Ma D, Dong X, Zang S, Ma R, Zhao P, Guo D, Dai J, Chen F, Ye J and Ji C: Aberrant expression and clinical correlation of Notch signaling molecules in breast cancer of Chinese population. Asia Pac J Clin Oncol 7: 385-391, 2011.

31. Sun DW, Zhang HD, Mao L, Mao CF, Chen W, Cui M, Ma R, Cao HX, Jing CW, Wang Z, et al: Luteolin inhibits breast cancer development and progression in vitro and in vivo by suppressing Notch signaling and regulating MiRNAs. Cell Physiol Biochem 37: 1693-1711, 2015.

32. Li L, Zhao F, Lu J, Li T, Yang H, Wu C and Liu Y: Notch-1 signaling promotes the malignant features of human breast cancer through NF-kappaB activation. PLoS One 9: e95912, 2014. 